Mini Review

\title{
Kidney biomarkers in hepatorenal syndrome
}

\begin{abstract}
Cirrhosis is the result of advanced liver disease characterized by fibrosis due to increased portal hypertension. Patients with cirrhosis are prone to develop acute kidney injury (AKI) triggered by bacterial infections, hypovolemia, nephrotoxic drugs and intrinsic kidney disease. The diagnostic criteria for the diagnosis of HRS continue to rely on serum creatinine levels which should be interpreted with caution in patients with cirrhosis. To date, accurate biomarkers indicative of renal function are lacking. Further validation with prospective studies is warranted to evaluate the value of novel biomarkers.
\end{abstract}

Keywords: hepatorenal syndrome, acute kidney injury, cirrhosis, biomarkers

\author{
Volume 7 Issue I - 2017
}

\author{
Natalia E Castillo,' Patricia Guzman Rojas, \\ Jan Freeman ${ }^{2}$ \\ 'Department of Internal Medicine, University of Central Florida \\ College of Medicine, USA \\ ${ }^{2}$ Department of Liver and Gastroenterology Unit, Royal Derby \\ Teaching Hospital, UK
}
Correspondence: Jan Freeman, Department of Liver and Gastroenterology Unit Royal Derby Teaching Hospital, Uttoxeter Road, Derby, UK, DE223N, Tel I33278723I, Email j.freeman115@btinternet.com

Received: May 01, 2017 | Published: June 22, 2017
Abbreviations: HRS, hepatorenal syndrome; IAC, international ascites club; GFR, glomerular filtration rate; AKI, acute kidney injury; SCr, serum creatinine; NGAL, neutrophil gelatinase associated lipocalin; KIM-1, kidney injury molecule 1; L-FABP, livertype fatty acid binding protein; TTF3, trefoil factor 3

\section{Introduction}

Hepatorenal syndrome (HRS) is a rapidly progressive functional form of acute renal failure. It occurs as a complication of advanced liver disease and is associated with significant mortality. ${ }^{1}$ Recurrent episodes of acute kidney injury are common in end-stage cirrhosis. Evaluation of kidney function and identification of kidney changes in patients with cirrhosis is crucial in predicting reversibility, treatment, and prognosis. In patients with cirrhosis, currently available noninvasive tools to assess renal function are inaccurate. Several novel biomarkers have recently emerged to help identify HRS from acute tubular necrosis (ATN) and enhance management and outcome.

\section{Discussion}

HRS was initially defined in 1996 by the International Ascites Club (IAC) Table 1. ${ }^{2}$ However, the IAC criteria had several limitations including a serum creatinine ( $\mathrm{SCr}$ ) of $\leq 1.5 \mathrm{mg} / \mathrm{dL}$. This creatinine cut off excludes physiological fluctuations and a true estimate of glomerular filtration rate (GFR). ${ }^{3}$ In 2015, the IAC proposed a new definition of acute kidney injury (AKI) in cirrhosis. AKI is defined as the abrupt loss of kidney function with a subsequent increase of $0.3 \mathrm{mg} / \mathrm{dl}$ in SCrin 48 hours or a $50 \%$ increase from baseline within 7 days. ${ }^{4}$ Data suggest that early detection of kidney failure may increase the probability of kidney regression and reduce mortality rates. ${ }^{5}$

Table I Initial diagnostic criteria for hepatorenal syndrome-1996

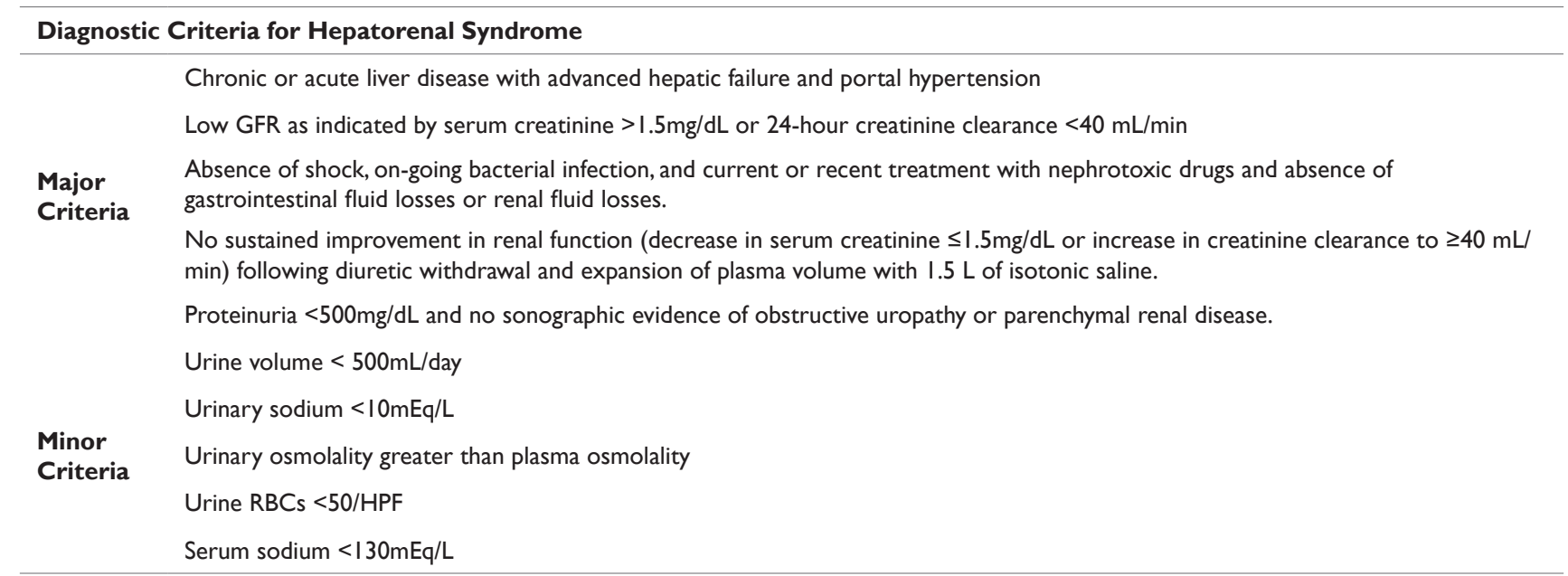

In patients with cirrhosis, $\mathrm{SCr}$ levels should be interpreted with caution. Creatinine is a marker of filtration and can be used to detect a decline in kidney function, but lacks specificity. Serum creatinine in patients with cirrhosis may be influenced by increased volume of distribution, reduced production of endogenous creatinine, muscle wasting, medications and elevated bilirubin. ${ }^{3,6}$ To date, the diagnosis of HRS is based solely upon clinical criteria and more discriminating tests are required to guide the allocation of treatment and to help predict progression of AKI. In this review, we discuss novel biomarkers of renal function that can improve diagnostic accuracy. 
Nearly 30 biomarkers of kidney tubular injury have been investigated for early detection, differential diagnosis and prognosis of AKI in cirrhosis. ${ }^{7}$ Ischemia-related events are a common mechanism in AKI that can lead to tubule injury. Cystatin $\mathrm{C}$ is a low molecular weight protein that is independent of sex, gender, inflammatory condition, and malignancy. ${ }^{8}$ Studies have suggested that serum cystatin $\mathrm{C}$ is a more sensitive marker of GFR than $\mathrm{SCr}$ since it freely crosses the glomerular membrane to be reabsorbed and metabolized in the proximal tubular cells, without being eliminated. ${ }^{7}$ Cystatin $\mathrm{C}$ has been also shown to be a predictor of HRS and mortality in patients with liver cirrhosis and ascites. ${ }^{9}$

Other tubular proteins unregulated by injury include Neutrophil Gelatinase-Associated Lipocalin (NGAL), Kidney injury molecule 1 (KIM-1), and Liver-type fatty acid binding protein (L-FABP). ${ }^{7-10}$ In animal models, NGAL is released in the urine following an ischemic or nephrotoxic insult. Human studies suggest that NGAL measurements either in urine and serum may differentiate acute tubular necrosis from type 1 HRS, pre-renal azotemia or chronic kidney disease. ${ }^{11}$ Two recent studies suggest that elevated urinary NGAL is predictive of early mortality in cirrhotic patients with AKI. ${ }^{12,13}$ However, NGAL lacks specificity as it is also elevated in other acute and chronic inflammatory conditions.

Kidney injury molecule 1 (KIM-1) is a transmembrane protein and a marker of proximal tubular injury. ${ }^{14}$ Few studies have monitored KIM-1 in patients with cirrhosis and AKI. However, KIM-1 levels have been shown to predict the development of HRS in patients with advanced cirrhosis. Interestingly, KIM-1 levels are not as accurate as NGAL, oteopontin, albumin and trefoil factor 3 (TTF3) for the diagnosis of ATN in patients with cirrhosis. ${ }^{15}$

Liver-type fatty acid binding protein (L-FABP) is a small protein that is also expressed in the proximal tubular epithelium in humans. ${ }^{16}$ In animal models, urinary L-FABPis elevated in kidney disease with tubule-interstitial damage. ${ }^{15}$ L-FABP has been evaluated in the diagnosis of AKI and sepsis complicated by AKI. ${ }^{5}$ The prognostic utility of L-FABP as a biomarker in HRS needs to be further elucidated.

Interleukin-18 (IL-18) is a marker released by recruited inflammatory cells. ${ }^{16}$ IL-18, in addition to other biomarkers (KIM1 and L-FABP), has been associated with progression of AKI and mortality in patients with cirrhosis. A recent meta-analysis showed that urine levels of IL-18 and NGAL are elevated in ATN compared to non-ATN AKI. ${ }^{17}$ IL-18 has also been described as a prognostic tool in patients with cirrhosis.

Activation and up regulation of toll like receptors (TLRs) may play a role in HRS. In AKI, necrotic tubular cells release TLR legends which could activate other tubular cells and other immune molecules in the kidney. High levels of TLR-4 have been found in patients with cirrhosis and AKI suggesting a potential role of TLR-4 as a mediator of renal injury. ${ }^{18}$ Further studies are needed to determine if urinary TLR-4 could help to diagnose AKI.

Other kidney biomarkers including osteopontin have been shown to be predictive of early mortality in intensive care unit patients with AKI. Osteopontin is a cytokine that is mainly expressed in the proximal and distal tubular cells. ${ }^{19}$ Serum osteopontin, NGAL and TFF-3 have been found to be associated with acute on chronic liver failure. Moreover, it has been linked to a 3-month survival in patients with acute decomposition of cirrhosis. ${ }^{15}$

\section{Conclusion}

Kidney impairment in cirrhosis occurs due to both systemic vasodilatation and inflammation leading to chronic kidney vasoconstriction. Currently available noninvasive tools to assess renal function are inaccurate in patients with cirrhosis. Novel kidney biomarkers (cystatin C, NGAL, IL-8, L-FABP and KIM-1) may be able to predict progression and mortality in patients with AKI and may be able to diagnose AKI earlier than a change in creative or estimated GFR. As additional markers become clinically available, clinicians should combine functional and structural biomarkers to more precisely elucidate the etiology and course to initiate prompt therapy in AKI in cirrhosis.

\section{Acknowledgments}

None.

\section{Conflicts of interest}

Authors declare that there is no conflict of interest.

\section{References}

1. Gines P, Schrier RW. Renal failure in cirrhosis. $N$ Engl Med. 2009;361(13):1279-1290.

2. Arroyo V, Gines P, Gerbes AL, et al. Definition and diagnostic criteria of refractory ascites and hepatorenal syndrome in cirrhosis. Hepatology. 1996;23(1):164-176.

3. Sherman DS, Fish DN, Teitelbaum I. Assessing renal function in cirrhotic patients: problems and pitfalls. Am J Kidney Dis. 2003;41(2):269-278.

4. Angeli P, Gines P, Wong F, et al. Diagnosis and management of acute kidney injury in patients with cirrhosis: revised consensus recommendations of the International Club of Ascites. Gut. 2015;62(4):531-537.

5. Belcher JM, Sanyal AJ, Peixoto AJ, et al. Kidney biomarkers and differential diagnosis of patients with cirrhosis and acute kidney injury. Hepatology. 2014;60(2):622-632.

6. Francoz C, Glotz D, Moreau R, et al. The evaluation of renal function and disease in patients with cirrhosis. J Hepatol. 2010;52(4):605-613.

7. Francoz C, Nadim MK, Durand F. Kidney biomarkers in cirrhosis. $J$ Hepatol. 2016;65(4):809-824.

8. Herget-Rosenthal S, Trabold S, Pietruck F, et al. Cystatin C: efficacy as screening test for reduced glomerular filtration rate. Am J Nephrol. 2000;(2):97-102.

9. Sharawey MA, Shawky EM, Ali LH, et al. Cystatin C: a predictor of hepatorenal syndrome in patients with liver cirrhosis. Hepatol Int. 2011;5(4):927-933.

10. Belcher JM. Acute Kidney Injury in Liver Disease: Role of Biomarkers. Adv Chronic Kidney Dis. 2015;22(5):368-375.

11. Mishra J, Ma Q, Prada A, et al. Identification of neutrophil gelatinaseassociated lipocalin as a novel early urinary biomarker for ischemic renal injury. J Am Soc Nephrol. 2003;14(10):2534-2543.

12. Barreto R, Elia C, Sola E, et al. Urinary neutrophil gelatinase-associated lipocalin predicts kidney outcome and death in patients with cirrhosis and bacterial infections. J Hepatol. 2001;61(1):35-42.

13. Verna EC, Brown RS, Farrand E, et al. Urinary neutrophil gelatinaseassociated lipocalin predicts mortality and identifies acute kidney injury in cirrhosis. Dig Dis Sci. 2012;57(9):2362-2370.

14. Han WK. Kidney Injury Molecule-1 (KIM-1): a novel biomarker for human renal proximal tubule injury. Kidney Int. 2002;62(1):237-244.

15. Ariza X, Sola E, Elia C, et al. Analysis of a urinary biomarker panel for clinical outcomes assessment in cirrhosis. PloS One. 2015;10(6):e0128145. 
16. Belcher JM, Garcia-Tsao G, Sanyal AJ, et al. Urinary biomarkers and progression of AKI in patients with cirrhosis. Clin J Am Soc Nephrol. 2014;9(11):1857-1867.

17. Jeremy Puthumana, Xavier Ariza, Justin M Belcher, et al. Urine Interleukin 18 and Lipocalin 2 Are Biomarkers of Acute Tubular Necrosis in Patients With Cirrhosis: A Systematic Review and Meta-Analysis Clinical Gastroenterology and Hepatology. 2016;15(7):1003-1003.e3.
18. Adebayo D, Morabito V, DavenportA, et al. Renal dysfunction in cirrhosis is not just a vasomotor nephropathy. Kidney Int. 2015;87(3):509-515.

19. Verstrepen WA, Persy VP, Verhulst A, et al. Renal osteopontin protein and mRNA upregulation during acute nephrotoxicity in the rat. Nephrol Dial Transplant. 2001;16(4):712-724. 